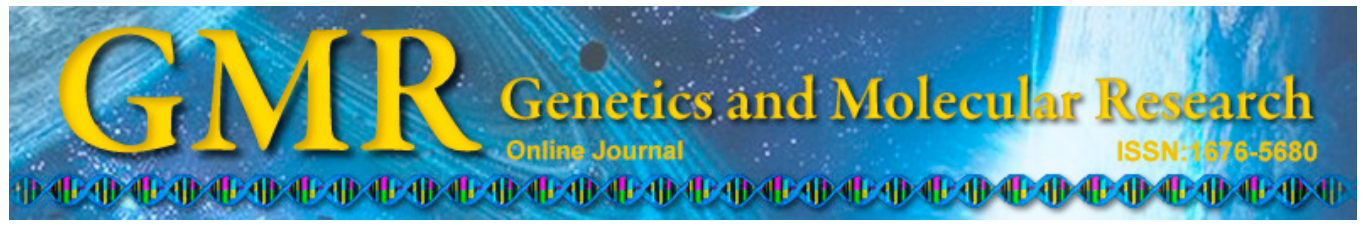

\title{
Intracellular survival of virulence and low-virulence strains of Vibrio parahaemolyticus in Epinephelus awoara macrophages and peripheral leukocytes
}

\author{
X.J. Xu1 ${ }^{1,2,3}$, B.H. Sang ${ }^{1,2,3}$, W.B. Chen ${ }^{1,2,3}$, Q.P. $\operatorname{Yan}^{1,2,3}$, Z.Y. Xiong ${ }^{1,2,3}$, \\ J.B. Su ${ }^{1,2}$ and W.Z. Zou ${ }^{1,2,3}$ \\ ${ }^{1}$ Fisheries College, Jimei University, Xiamen, Fujian, China \\ ${ }^{2}$ Key Laboratory of Healthy Mariculture for the East China Sea, \\ Ministry of Agriculture, Jimei University, Xiamen, Fujian, China \\ ${ }^{3}$ Fujian Provincial Key Laboratory of Marine Fishery Resources and \\ Eco-Environment, Jimei University, Xiamen, Fujian, China \\ Corresponding author: Q.P. Yan \\ E-mail: yanqp@jmu.edu.cn
}

Genet. Mol. Res. 14 (1): 706-718 (2015)

Received January 3, 2014

Accepted May 1, 2015

Published January 30, 2015

DOI http://dx.doi.org/10.4238/2015.January.30.14

\begin{abstract}
In this study, we examined the virulence factors and pathogenesis of Vibrio parahaemolyticus in Epinephelus awoara. The chemotactic motility of $V$. parahaemolyticus for phagocytosis and intracellular survival in fish macrophages was determined using virulence strains and low-virulence strains of $V$. parahaemolyticus. We found that the intracellular mean number of virulence strains of $V$. parahaemolyticus ranged from 0-180 min after co-incubation with macrophages and peripheral leukocytes, was relatively low, and decreased steadily over the observation period. Low-virulence strains of $V$. parahaemolyticus were unable to survive in peripheral leukocytes and macrophages. Cell viability in response to $V$. parahaemolyticus was assessed using the MTT assay. Low-virulence $V$. parahaemolyticus strains exhibited lower cytotoxicity compared to virulent strains.
\end{abstract}


The average percent of live macrophages and peripheral leukocytes infected by $V$. parahaemolyticus ranged from $13.50-79.20 \%$. These results indicate that $V$. parahaemolyticus in E. awoara is a facultative intracellular bacterium that may be involved in virulence.

Key words: Epinephelus awoara; Intracellular survival; Macrophages; Peripheral leukocytes; Vibrio parahaemolyticus

\section{INTRODUCTION}

Epinephelus awoara is an important marine fish farmed in China, particularly in the Guangdong and Fujian Provinces. With the rapid development of the E. awoara in the aquaculture industry, infectious diseases caused by bacteria have increased, leading to large economic losses. Vibrio parahaemolyticus has emerged as a major pathogen that infects grouper and other marine fish in aquaculture worldwide (Rodríguez et al., 2006; Chakraborty and Surendran, 2008; Blackwell and Oliver, 2008). The relationship between $V$. parahaemolyticus and fish has been widely recognized (Woo et al., 2006). Pathogens that can survive and persist, express a broad array of virulence that functions to circumvent the host immune system (Pawaria et al., 2007; Li et al., 2008; Roop et al., 2009). Macrophages and neutrophil granulocytes are the primary host cells for Vibrio species. When phagocytic cells ingest bacteria, they wrap the bacteria with their plasma membrane and incorporate the newly formed phagosomes into the cell. Several Vibrio species, including V. parahaemolyticus, can invade and replicate inside immune system cells and are specialized for ingesting and killing microorganisms.

Pathogen invasion is initiated by binding of the pathogen to the host cell surface followed by internalization. The intracellular fate of invading bacteria can vary greatly. Some invasive bacteria appear to survive intracellularly for extended periods. Elimination of intracellular Vibrio depends on activation of antimicrobial effector mechanisms in infected macrophages (Abd et al., 2005).

The purpose of this study was to examine the interaction between $V$. parahaemolyticus and phagocytic cells of E. awoara, a cell type likely to interact with natural infection. This cell may be a convenient cell model for assessing the ingestion and intracellular survival of $V$. parahaemolyticus and for analyzing the role of these bacterial strains in causing diseases in E. awoara.

\section{MATERIAL AND METHODS}

\section{Fish}

Approximately 500-600 g healthy E. awoara per individual were obtained from the Jimei Market, Xiamen.

\section{Bacterial strains and culture conditions}

In this study, 2 strains of $V$. parahaemolyticus with different virulences (a virulence strain and a low-virulence strain) were used (Huang et al., 2006). V. parahaemolyticus were grown on marine agar $2216 \mathrm{E}$ (Yi et al., 2008) at $28^{\circ} \mathrm{C}$ for $18 \mathrm{~h}$. The number of bacteria was adjusted to approximately $1.4 \times 10^{9}$ colony-forming units (CFU)/mL. 


\section{In vivo experiment}

Six E. awoara weighing approximately $500 \mathrm{~g}$ were obtained from Jimei Market, Xiamen. All fish used in this study appeared to be healthy before the experiments were carried out. Parasites were not identified on wet mount preparations prepared from the skin and gills. V. parahaemolyticus was not isolated on marine agar $2216 \mathrm{E}$ from samples obtained from the skin, gill, kidney, and spleen. Individuals from the infected group were intraperitoneally injected with $1 \times 10^{9} \mathrm{CFU}$ virulent $V$. parahaemolyticus in $0.2 \mathrm{~mL} 0.85 \%$ sterile saline solution. Six E. awoara were intraperitoneally injected with $0.2 \mathrm{~mL} 0.85 \%$ sterile saline solution without any bacteria and served as non-infected controls. The 2 groups were raised in two 10-L aquariums supplied with filtered and aerated seawater at $28^{\circ} \mathrm{C}$. Three days postinoculation, 6 E. awoara were dead. The low-virulence strain was injected into another 6 healthy E. awoara. The methods were the same as those described above. Disease signs and mortality in E. awoara were monitored after 3 days post-inoculation. All fish were sampled for bacteriological examination. The experiment was conducted 3 times.

\section{Macrophage and peripheral leukocyte isolation and culture}

\section{Macrophage isolation and culture}

The E. awoara were rinsed with alcohol and under aseptic conditions, the tissue overlaying the pronephros of each side was removed, and the organ was removed. Both head kidneys were pushed through a 100-order nylon net and suspended in $2 \mathrm{~mL}$ ice-cold Leibovitz L-15 medium (Biological Industries, Beit-Haemek, Israel) supplemented with 10 IU heparin/ $\mathrm{mL}$ and $2 \%$ fetal calf serum (FCS). Using a syringe plunger, ice-cold L-15 medium and heparin was added dropwise to wash the cells into a siliconized glass container on ice. The suspensions were then loaded onto a 34/51\% discontinuous Percoll (Amersham Pharmacia Biotech, Buckinghamshire, England, UK) density gradient and centrifuged at $350 \mathrm{~g}$ for $35 \mathrm{~min}$ at $4^{\circ} \mathrm{C}$. The band of cells at the interface of the Percoll gradient was collected, and then washed twice at $2000 \mathrm{r} / \mathrm{min}$ for $10 \mathrm{~min}$ at $4^{\circ} \mathrm{C}$. Isolated macrophages were resuspended in L-15 medium containing 5\% FCS, $100 \mathrm{IU}$ streptomycin/penicillinase $(\mathrm{S} / \mathrm{P}) / \mathrm{mL}$, and $10 \mathrm{IU}$ heparin $/ \mathrm{mL}$ and adjusted to a concentration of $2.4 \times 10^{7}$ cells $/ \mathrm{mL}$. The cells were distributed ( $1 \mathrm{~mL}$ per well) into the wells of a 6-well tissue culture plate (Corning, Inc., Corning, NY, USA). The numbers of living and dead cells were counted using the trypan blue method.

\section{Peripheral leukocyte isolation and culture}

Blood was collected from the tail vein of healthy E. awoara using $0.1 \%(\mathrm{v} / \mathrm{v})$ heparin sodium as an anticoagulant. E. awoara was bled as completely as possible using a syringe and the blood was placed in ice-cold L-15 medium containing $0.1 \% \mathrm{FCS}, 100 \mathrm{IU} \mathrm{S} / \mathrm{P} / \mathrm{mL}$, and $10 \mathrm{IU}$ heparin/mL. The samples were thoroughly mixed in a 1:1 ratio. Cell suspensions were then loaded onto a $60 \%$ Percoll density gradient using a syringe and centrifuged at 350 $g$ for $15 \mathrm{~min}$ at $4^{\circ} \mathrm{C}$. The band of cells at the interface of the Percoll gradient was collected. The process was repeated. 


\section{Assessment of viable intracellular $V \cdot$ parahaemolyticus}

\section{Cell invasion assays}

Peripheral leukocytes and macrophages from the E. awoara head kidney were seeded on 6-well tissue culture plates at $1 \mathrm{~mL} /$ well before the bacteria were added. $V$. parahaemolyticus was used to infect peripheral leukocytes and macrophages at $5 \times 10^{8} \mathrm{CFU} /$ well. Bacterial cell suspensions of $V$. parahaemolyticus were inoculated onto peripheral leukocytes and macrophages at a 1:100 multiplicity of infection (MOI); $1 \mathrm{~mL}$ of the bacterial suspension was added to each well. Infected cells were incubated at $28^{\circ} \mathrm{C}$ in $5 \% \mathrm{CO}_{2}$ to allow for bacterial entry. After $2 \mathrm{~h}$ of incubation, the cells were poured into sterile, siliconized tubes. The supernatant was carefully withdrawn to avoid disturbing the packed cells and the supernatant was discarded. The infected cells were then washed with $3 \mathrm{~mL}$ phosphate-buffered saline (PBS; $\mathrm{pH}$ 7.0), and again centrifuged for $5 \mathrm{~min}$ at $100 \mathrm{~g}$ at $28^{\circ} \mathrm{C}$. The mixture was washed twice using the same method. After discarding the supernatant, the washed cells were resuspended in $2 \mathrm{~mL}$ PBS; cell-free extracellular bacteria were separated from cell-associated intracellular bacteria by centrifugation at $100 \mathrm{~g}$ for $5 \mathrm{~min}$ at $28^{\circ} \mathrm{C}$. The pellet containing cell-associated microorganisms was treated with $2000 \mathrm{U} \mathrm{S} / \mathrm{P} / \mathrm{mL}$ for $20 \mathrm{~min}$ at $28^{\circ} \mathrm{C}$ to kill the extracellular bacteria in invasion assay. To eliminate gentamicin, infected cells were washed twice with PBS as described above. The upper half of the supernatant fluid was withdrawn and plated on beef extract-peptone agar. Fresh L-15 medium with $10 \mathrm{IU}$ heparin/mL, 5\% FCS, and $100 \mathrm{U}$ $\mathrm{S} / \mathrm{P}$ were added. To estimate changes in the mean number of viable intracellular $V$. parahaemolyticus in peripheral leukocytes and macrophages, this time was enacted as zero. Intracellular bacteria were quantified by plating serial dilutions of the lysate. All quantitative invasion assays were performed in triplicate. A noninvasive strain of $V$. parahaemolyticus was used as a negative control.

\section{Intracellular growth assay}

The cells that had engulfed the bacteria continued to be cultured at $28^{\circ} \mathrm{C}$ in $5 \% \mathrm{CO}_{2}$ for $0,30,60,120$, and $180 \mathrm{~min}$. At each time point, the cells were centrifuged for $5 \mathrm{~min}$ at $100 \mathrm{~g}$ at $28^{\circ} \mathrm{C}$ and the supernatant was aspirated; next, $1 \mathrm{~mL}$ sterile distilled water was added and the mixture was incubated for $30 \mathrm{~min}$, followed by cell lysis. The cell lysate from these time points was acquired in triplicate by plating dilutions of the sample onto antibiotic-free beef extractpeptone agar, incubating at $28^{\circ} \mathrm{C}$ for $48 \mathrm{~h}$; intracellular bacteria were quantified over time and bacterial titers were determined by serial dilution and plating. The $\log _{10}$ change in the CFU of the V. parahaemolyticus/well was then calculated. Experiments were conducted in triplicate.

\section{Assessment of cell viability}

The viability of macrophages and peripheral leukocytes was determined using an MTT [3-(4,5-dimethylthiazol-2-yl)-2,5-diphenyltetrazolium bromide] assay as described previously (Düchler and Stepnik, 2008). Macrophages and peripheral leukocytes were seeded separately on 96-well culture plates at $1.0 \times 10^{5}$ cells per well containing $100 \mu \mathrm{L} \mathrm{L}-15$ medium and 5\% FCS. The cells were allowed to adhere to the plate for $2 \mathrm{~h}$ in $5 \% \mathrm{CO}_{2}$ and then non-adherent cells were removed. Adherent cells were used for infection assays and cultured in L-15 
medium containing 5\% FCS without antibiotics. Macrophages and peripheral leukocytes were incubated in the presence of 5\% serum with virulence strains and low-virulence strains of $V$. parahaemolyticus in 50:1, 100:1, 500:1, and 1000:1 MOI for $1 \mathrm{~h}$. After incubation with drugs in $5 \%$ serum for $1 \mathrm{~h}$ at a cell density of $1.0 \times 10^{5}$ cells per well, macrophages and peripheral leukocytes were washed 3 times in sterile PBS and resuspended in fresh L-15 medium containing $100 \mathrm{U} \mathrm{S}-\mathrm{P} / \mathrm{mL}, 10 \mathrm{IU}$ heparin $/ \mathrm{mL}$, and 5\% FCS. The medium was removed and $20 \mu \mathrm{L}$ $(5 \mathrm{mg} / \mathrm{mL})$ MTT was added to each well. The cells were incubated in a humidified atmosphere of $5 \% \mathrm{CO}_{2}$ at $28^{\circ} \mathrm{C}$ for $4 \mathrm{~h}$. After incubation, the supernatant from each well was carefully removed and the dark crystals formed were dissolved by adding $100 \mu \mathrm{L}$ dimethyl sulfoxide to each well. Subsequently, plates were incubated for $30 \mathrm{~min}$ and the optical densities at 570 $\mathrm{nm}$ were measured by transferring $100-\mu \mathrm{L}$ aliquots to 96 -well plates and using a plate reader with a corresponding filter. Data are reported as the percentage of the value obtained from cells incubated in fresh medium only. The percentage of viable cells was defined as [(experimen-

tal $_{\text {absorbance }}-$ blank $\left._{\text {absorbance }}\right) /$ control $_{\text {absorbance }}-$ blank $\left.\left._{\text {absorbance }}\right)\right] \times 100$, where the control $l_{\text {absorbance }}$ was the value obtained for $1.0 \times 10^{5}$ cells /well, which was the number plated at the beginning of the experiment, and blank absorbance $_{\text {was }}$ the value obtained in wells containing medium and MTT without cells. Assays were repeated 3 times.

\section{Statistical analysis}

The results are reported as means \pm standard deviation. The Student $t$-test following one-way analysis of variance was used to evaluate the significance of differences. P values less than 0.05 were considered to be significant.

\section{RESULTS}

\section{In vivo experiments}

Fish in the negative control group (injected with $0.2 \mathrm{~mL} 0.85 \%$ sterile saline solution without any bacteria) exhibited no clinical signs or mortality signs (Figure 1). All fish in the infected group that had been inoculated intraperitoneally with $V$. parahaemolyticus exhibited mortality and showed clinical symptoms of infection beginning on 2 post-inoculation (Figure 2). Infected fishes died between days 2 and 3. Upon necropsy, the fishes showed abdominal intumescentia, anal red swelling, and other typical external signs. Abdomens from affected fish were distended because of fluid accumulation. Thus, the pathogenic bacterium V. parahaemolyticus was found to have infected E. awoara. The virulence strains included highly virulent and low-virulence strains, which showed relatively low virulence towards E. awoara in this in vivo experiment.

\section{Collection of peripheral leukocytes and macrophages}

Approximately $10^{8}$ cells $/ \mathrm{mL}$ macrophages were harvested from the head kidney of $E$. awoara. At least $10^{8}$ cells $/ \mathrm{mL}$ peripheral leukocytes were collected from the tail vein blood of E. awoara. The viability of the phagocytes, based on trypan blue exclusion, exceeded $98 \%$ in all cases. Upon light microscopical examination, the rounded cells that adhered onto the cover slips consisted of $80 \%$ phagocytes and $20 \%$ lymphocytes. 


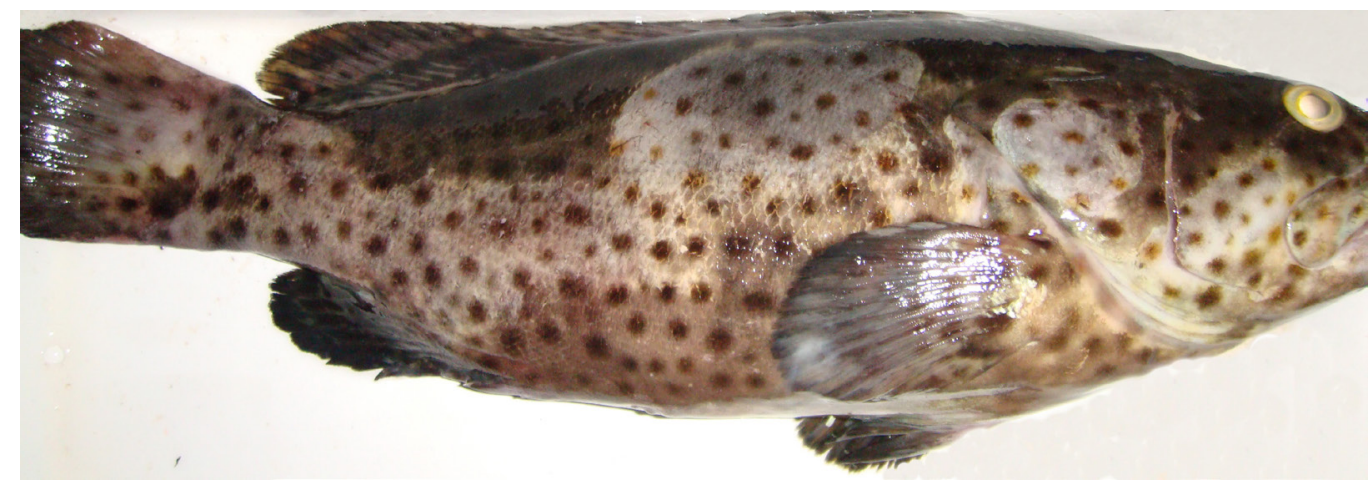

Figure 1. Healthy Epinephelus awoara exhibited no clinical symptoms.

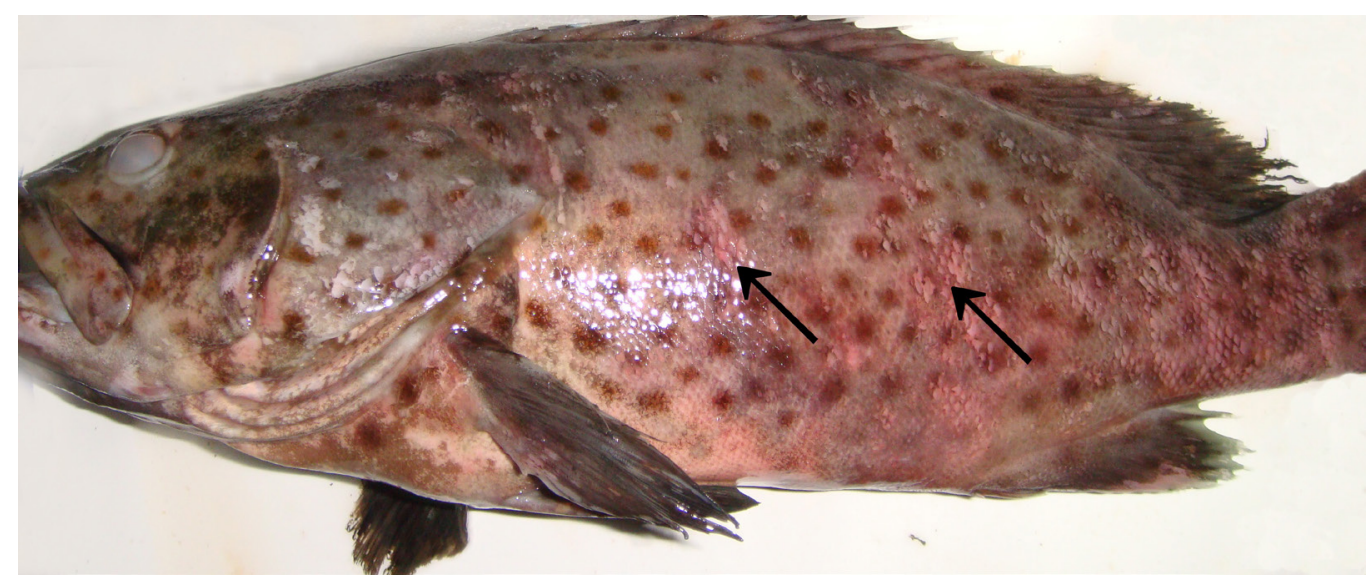

Figure 2. Typical external sign of Epinephelus awoara challenged with Vibrio parahaemolyticus showing red swelling (arrows).

\section{Intracellular survival of $\boldsymbol{V}$. parahaemolyticus in macrophages and peripheral leukocytes}

The chemotactic motility of $V$. parahaemolyticus for phagocytosis and intracellular survival in fish macrophages was determined using virulent strains and low-virulence strains of $V$. parahaemolyticus. The ability of $V$. parahaemolyticus to enter peripheral leukocytes and macrophages of E. awoara prompted us to examine their intracellular fates. Intracellular growth assays were performed using $V$. parahaemolyticus-infected peripheral leukocytes and macrophages. The intracellular mean number of CFU of $V$. parahaemolyticus per well of tissue culture plates at different times of re-incubation of infected peripheral leukocytes or macrophages is shown Table 1. Before re-incubation, the mean number of intracellular CFU of $V$. parahaemolyticus in macrophages and peripheral leukocytes per well was $338 \pm 8.84 \mathrm{x}$ $10^{5} \mathrm{CFU} /$ well and $54.17 \pm 5.13 \times 10^{5} \mathrm{CFU} /$ well, respectively. During the first $30 \mathrm{~min}$ of reincubation, the mean number of viable intracellular bacteria in macrophages and peripheral leukocytes was approximately twice the value observed before re-incubation. 


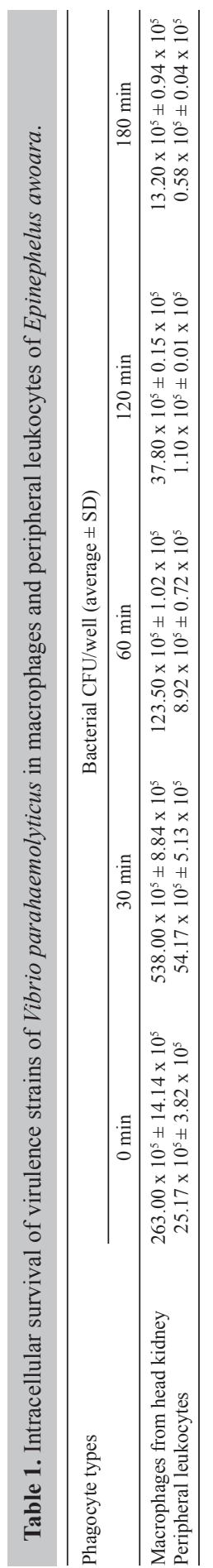


After $60 \mathrm{~min}$ of re-incubation, the intracellular mean number of CFU $V$. parahaemolyticus decreased. After 60 min of re-incubation, the mean numbers of viable intracellular bacteria in macrophages and peripheral leukocytes were 77 and $84 \%$ of the mean numbers observed after $30 \mathrm{~min}$ of re-incubation. After $120 \mathrm{~min}$ of re-incubation, however, the number of viable bacteria decreased. After $180 \mathrm{~min}$ of re-incubation, viable bacteria could no longer be detected.

Differences in the mean number of viable intracellular bacteria between tests performed using low-virulence strains of $V$. parahaemolyticus and tests performed using virulent strains of $V$. parahaemolyticus are shown in Table 2 . The data were statistically significant at 0 , $30,60,120$, and $180 \mathrm{~min}$ of re-incubation. For all strains, the numbers of intracellular bacteria were relatively low and decreased progressively during the observation period.

Low-virulence strains of $V$. parahaemolyticus could not survive in peripheral leukocytes and macrophages. Values were significantly different $(\mathrm{P} \leq 0.05)$.

Table 2. Intracellular survival of low-virulence strains of Vibrio parahaemolyticus in macrophages and peripheral leukocytes of Epinephelus awoara.

\begin{tabular}{lccccc}
\hline Phagocyte types & \multicolumn{4}{c}{ Bacterial CFU/well (average \pm SD) } \\
\cline { 2 - 5 } & 0 min & 30 min & 60 min & $120 \mathrm{~min}$ & $180 \mathrm{~min}$ \\
\hline Macrophages from head kidney & 0 & 0 & 0 & 0 & 0 \\
Peripheral leukocytes & 0 & 0 & 0 & 0 \\
\hline
\end{tabular}

\section{Cell viability assays: reduction of formazan dye (MTT)}

To determine the involvement of $V$. parahaemolyticus in macrophage and peripheral leukocyte death, $V$. parahaemolyticus were inoculated with peripheral leukocytes and macrophages at of 50:1, 100:1, 500:1, and 1000:1 MOI. Cell viability in response to V. parahaemolyticus was assessed in peripheral leukocytes and macrophages using the MTT assay. The results of the cytoxicity assay are shown in Figures 3 and 4. V. parahaemolyticus was cytotoxic towards macrophages and peripheral leukocytes, with the low-virulence strains of $V$. parahaemolyticus showing lower cytotoxicity in macrophages (Figure 3) and peripheral leukocytes (Figure 4) compared to virulent strains. During the experiment, the average percent of live macrophages and peripheral leukocytes infected with low-virulence strains of $V$. parahaemolyticus was statistically higher than cell survival of the cells infected with virulence strains of $V$. parahaemolyticus at various MOIs (Figures 3 and 4). However, the percentage of live macrophages and peripheral leukocytes infected with $V$. parahaemolyticus decreased from 50:1 to 1000:1 MOI. Virulent strains of $V$. parahaemolyticus were cytotoxic for peripheral leukocytes, showing a $63.6 \%$ loss in viability at of 50:1 MOI. Low-virulence strains were much less cytotoxic, but an approximately $79.2 \%$ loss of viability at the same MOI was observed. The virulence strains and low-virulence strains incubated with peripheral leukocytes retained 13.5 and $17.5 \%$ at $1000: 1 \mathrm{MOI}$, respectively.

Compared with peripheral leukocytes, the viability of macrophages infected with $V$. parahaemolyticus showed similar results (Figure 4). The average percent of live macrophages infected by low-virulence strains of $V$. parahaemolyticus was $64.6 \%$ compared to $63.8 \%$ by virulence strains.

At 1000:1 MOI, the percent of macrophages infected with low-virulence strains was 
$17.7 \%$, while that infected with virulence strains decreased to $14.4 \%$. Values were significantly different $(\mathrm{P} \leq 0.05)$.

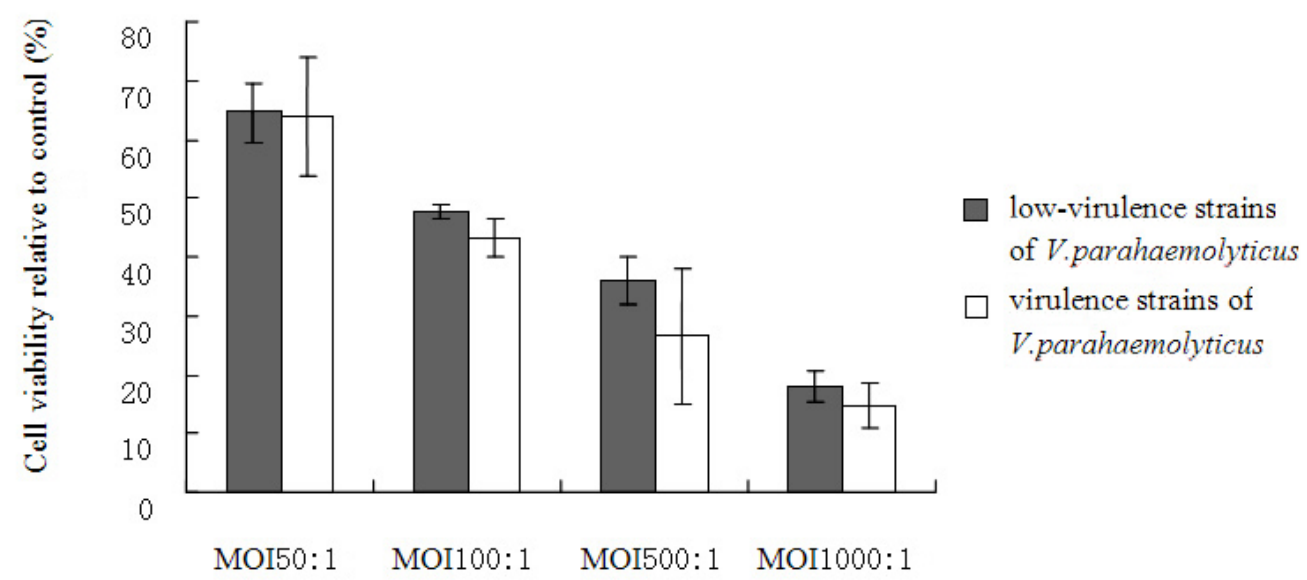

Figure 3. MTT test for cell survival of macrophages at different MOIs of virulence and low-virulence strains of Vibrio parahaemolyticus. Two independent experiments were performed in triplicate $(* \mathrm{P}<0.05$, analysis of variance, Tukey-Kramer).

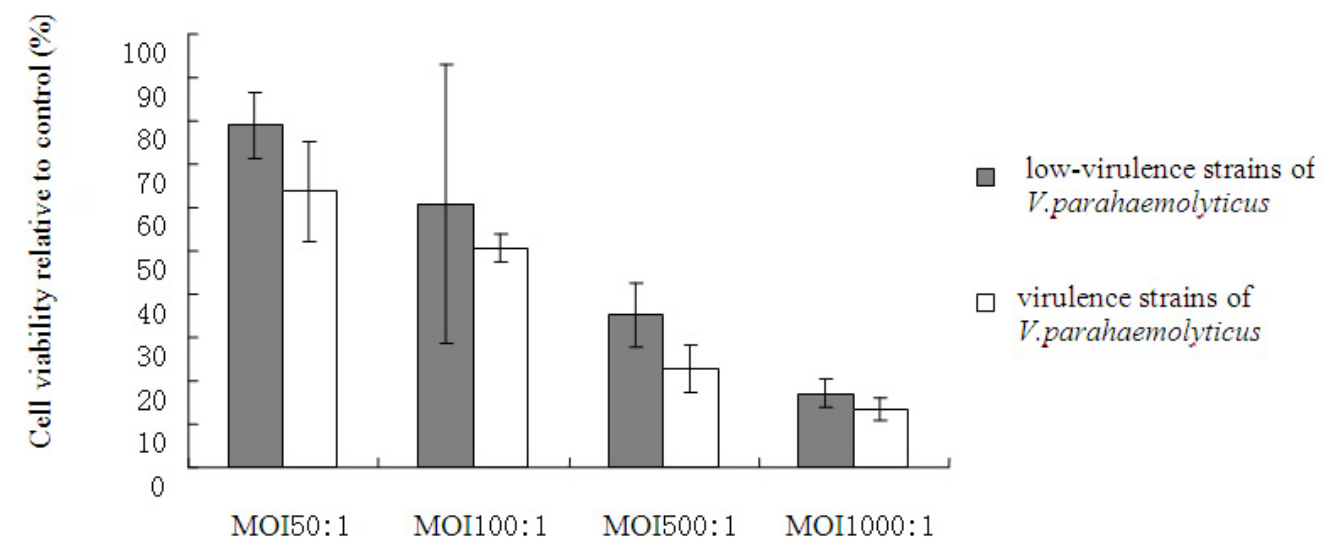

Figure 4. MTT test for cell survival of peripheral leukocytes at different MOIs of virulence and low-virulence strains of Vibrio parahaemolyticus. Two independent experiments were performed in triplicate $(* \mathrm{P}<0.05$, analysis of variance, Tukey-Kramer).

\section{DISCUSSION}

In vivo experiments showed that $V$. parahaemolyticus could be detected and identified as pathogenic bacteria in E. awoara. This is the first report describing the ability of $V$. parahaemolyticus to enter and survive intracellularly in cultured peripheral leukocytes and macrophages from E. awoara. Macrophages and peripheral leukocytes play an important role in the early immune response by killing invading microorganisms through phagocytosis and releasing bactericidal substances such as reactive oxygen species (Koh et al., 2010; Qiao et al., 
2013). Pathogens are likely to encounter phagocytic cells soon after entering the host. Phagocytic cells primarily act as effector cells in the immune response. Thus, it is not surprising that some pathogens have adapted to live inside macrophages and peripheral leukocytes for part of their life cycles, using the cell as a shield against other cell-mediated and humoral immune responses. Intracellular survival is a key part in the pathogenic cycle of a number of bacterial pathogens. The mechanisms by which intracellular pathogens resist being killed by peripheral leukocytes and macrophages include inhibition of phagosome-lysosome fusion, escape into the cytoplasmic compartment, and resistance to reactive oxygen intermediates and lysosomal enzymes (Ryckaert et al., 2010). Resistance to nonoxidative killing is indicated by the ability of $V$. parahaemolyticus to cause invasive infections in patients with disease, an inherited disorder of phagocytes in which peripheral leukocytes and macrophages are unable to generate microbicidal oxygen radicals (Ricardo et al., 2007; Düchler and Stepnik, 2008; Dean et al., 2009; Zhao et al., 2009; Mussá et al., 2012).

Currently, although the uptake of vibrio by macrophages has been characterized in detail, no comprehensive study of peripheral leukocytes and macrophages from E. awoara had been carried out. The importance of the interactions between peripheral leukocytes and macrophages with $V$. parahaemolyticus is clear.

In this study, we established a peripheral leukocyte and macrophage model of invasion and intracellular survival for $V$. parahaemolyticus. The mean number of viable intracellular bacteria was determined from 0-180 min and the data were found to be statistically significant. The numbers of intracellular $V$. parahaemolyticus were relatively low and decreased steadily throughout the experimental period. V. parahaemolyticus was capable of invading and surviving within macrophages and peripheral leukocytes in vitro. However, in contrast with the virulence strain, the low-virulence strain of $V$. parahaemolyticus was unable to survive intracellularly. Our findings provide further evidence that $V$. parahaemolyticus is a facultative intracellular pathogen that can invade and persist in peripheral leukocytes and macrophages. Our data clearly suggest that intracellular survival contributes to the virulence potential of pathogenic strains of $V$. parahaemolyticus. Differences in the ability of virulence and low-virulence strains of $V$. parahaemolyticus to survive intracellularly may facilitate our understanding of the varied disease progressions related to $V$. parahaemolyticus infections in E. awoara. Mussá et al. (2012) showed that phagocytosis resistance is a virulence mechanism of Haemophilus parasuis on porcine alveolar macrophages. Although the Nagasaki strain entered the cells, a negligible association with porcine alveolar macrophages was observed (Olvera et al., 2009).

We found that the invasion numbers of $V$. parahaemolyticus in cultured macrophages were significantly greater than those in peripheral leukocytes, suggesting that distinct or additional mechanisms mediate $V$. parahaemolyticus entry into cultured macrophages. One of the principal mechanisms of macrophage uptake was phagocytosis, which involves the uptake of particles coated with complement proteins and/or antibodies. Binding of complement proteins to the intracellular pathogens $V$. parahaemolyticus facilitates their entry into phagocytic cells (Zhao et al., 2009; Qiao et al., 2013).

The ability of $V$. parahaemolyticus to invade cultured macrophages and peripheral leukocytes and survive intracellularly clearly suggests that $V$. parahaemolyticus has an intracellular phase during infection in E. awoara. Intracellular survival in macrophages may play a role in immune evasion. Alternatively, macrophages and peripheral leukocytes may serve as a 
vehicle for the translocation and systemic dissemination of $V$. parahaemolyticus in E. awoara. Using our established cell culture model for invasion and intracellular survival, studies can be conducted to further characterize the mechanisms of pathogenesis and the genetic elements required for these processes. Finally, comparison of the behavior of virulence and low-virulence strains of $V$. parahaemolyticus in phagocytic cells using this model may provide additional information regarding the bacterial genes necessary for invasion and survival in different hosts.

In the present study, macrophages and peripheral leukocytes from fish were used to study the cytotoxicity of vibrio because of their well-defined culturing characteristics under experimental conditions and their relevance as an in vitro system for screening purposes. The MTT assay was used to determine cytotoxicity by evaluating the activity of mitochondrial enzyme dehydrogenase succinate. Applications of this method included testing of drug sensitivity, cytotoxicity, and cell viability. Other bacterial fish pathogens have been shown to survive inside macrophages, including Burkholderia pseudomallei, Burkholderia thailandensis, Burkholderia oklahomensis, and Piscirickettsia salmonis (Pan et al., 2010; Wand et al., 2011).

A recent study by Rajas et al. (2009) indicated that $P$. salmonis survived and replicated inside the macrophage/monocyte cell line RTS11 derived from rainbow trout spleen without inducing a cell death response. This led to the persistence of the bacteria and consequently their dissemination to other tissues, enabling evasion of the first line of defense against pathogens. In contrast, other bacteria, such as $B$. pseudomallei and B. thailandensis, had a cytotoxic effect on macrophages after uptake (Charoensap et al., 2009; Dean et al., 2009).

The cytotoxic effect of $V$. parahaemolyticus on peripheral leukocytes and macrophages observed in this study led to the death of phagocytes. Measurement of cell viability is valuable in a wide range of research areas. These experimental results demonstrated that the $V$. parahaemolyticus had cytotoxic effects on macrophages and peripheral leukocytes of $E$. awoara. The virulence strains of $V$. parahaemolyticus showed more cytotoxicity towards macrophages and peripheral leukocytes than did the low-virulence strains, which helped bacterial pathogens to release more virulence factors and caused host damage in vivo against the cellular immune defense. This activity of virulence strains may be related to the stronger resistance to macrophage-mediated killing through its higher superoxide dismutase activity (Qiao et al., 2013). Therefore, the ability to survive and replicate within macrophages is a common characteristic of virulence among several pathogenic bacteria.

Although the virulence strain was more resistant to macrophage-mediated killing activity than the low-virulence strain (Thirunavukkarasu et al., 2008), H. ducreyi persisted in dendritic cells without affecting eukaryotic cell viability (Banks et al., 2007). Therefore, the ability to survive inside macrophages appears to depend on the bacterial species. Different mechanisms may have evolved in different bacterial species to overcome the phagocyte-mediated defense system. Hence, resistance to macrophage-mediated bacterial killing plays an important role in the pathogenesis of $V$. parahaemolyticus.

Overall, the results presented in this study showed that in vitro studies of macrophages and peripheral leukocytes will help increase the understanding of the complex relationship between bacteria and the relationships among different pathogens in co-infection. Our in vitro studies enabled investigation of new pathways for the stimulation of the immune system to better respond to pathogens. In conclusion, we report immunological differences among virulence $v$ s low-virulence $V$. parahaemolyticus strains in their interactions with macrophages and peripheral leukocytes and their modulation by infection. 


\section{ACKNOWLEDGMENTS}

Research supported by the Science Foundation of the Fujian Province, China (\#2013J01140), the National Natural Science Foundation of China (General Program \#40976096), the Fujian Provincial Key Laboratory of Marine Fishery Resources and Eco-Environment (\#ZK2014004), and the Science Foundation of the Jimei Province (\#20122C01).

\section{REFERENCES}

Abd H, Weintraub A and Sandström G (2005). Intracellular survival and replication of Vibrio cholerae O139 in aquatic free-living amoebae. Environ. Microbiol. 7: 1003-1008.

Banks KE, Humphreys TL, Li W, Katz BP, et al. (2007). Haemophilus ducreyi partially activates human myeloid dendritic cells. Infect. Immun. 75: 5678-5685.

Blackwell KD and Oliver JD (2008). The ecology of Vibrio vulnificus, Vibrio cholerae, and Vibrio parahaemolyticus in North Carolina Estuaries. J. Microbiol. 7: 146-153.

Chakraborty RD and Surendran PK (2008). Occurrence and distribution of virulence strains of Vibrio parahaemolyticus in seafoods marketed from Cochin (India). World J. Microbiol. Biotechnol. 24: 1929-1935.

Charoensap J, Utaisincharoen P, Engering A and Sirisinha S (2009). Differential intracellular fate of Burkholderia pseudomallei 844 and Burkholderia thailandensis UE5 in human monocyte-derived dendritic cells and macrophages. BMC Immunol. 10: 20.

Dean RE, Ireland PM, Jordan JE, Titball RW, et al. (2009). RelA regulates virulence and intracellular survival of Francisella novicida. Microbiology 155: 4104-4113.

Düchler M and Stepnik M (2008). Cytotoxic effects of a combination of three natural compounds to leukemia cells in vitro. Cancer Ther. 6: 733-740.

Huang RF, Zhuang ZX, Yan QP, Li JX, et al. (2006). Quantitative detection of marine pathogenic bacteria using protein microarray immunoassay. Chinese J. Anal. Chem. 34: 1411-1414.

Koh MJ, Lee HS, Rhee JE and Choi SH (2010). Evidence that Vibrio vulnificus ahpC2 is essential for survival under high salinity by modulating intracellular level of ROS. J. Microbiol. 48: 129-133.

Li L, Michel R, Cohen J, Decarlo A, et al. (2008). Intracellular survival and vascular cell-to-cell transmission of Porphyromonas gingivalis. BMC Microbiol. 1: 1-11.

Mussá T, Rodríguez-Cariño C, Sánchez-Chardi A, Baratelli M, et al. (2012). Differential interactions of virulent and non-virulent $H$. parasuis strains with naïve or swine influenza virus pre-infected dendritic cells. Vet. Res. 43: 80-93.

Olvera A, Ballester M, Nofrarías M, Sibila M, et al. (2009). Differences in phagocytosis susceptibility in Haemophilus parasuis strains. Vet. Res. 40: 24.

Pan X, Tamilselvam B, Hansen EJ, Daefler S, et al. (2010). Modulation of iron homeostasis in macrophages by bacterial intracellular pathogens. BMC Microbiol. 10: 1471-1484.

Pawaria S, Rajamohan G, Gambhir V, Lama A, et al. (2007). Intracellular growth and survival of Salmonella enterica serovar Typhimurium carrying truncated hemoglobins of Mycobacterium tuberculosis. Microb. Pathog. 42: 119-128.

Qiao G, Jang IK, Won KM, Woo SH, et al. (2013). Pathogenicity comparison of high- and low-virulence strains of Vibrio scophthalmus in olive flounder Paralichthys olivaceus. Fish Sci. 79: 99-109.

Rajas V, Galanti N, Bols NC and Marshall SH (2009). Productive infection of Piscirickettsia salmonis in macrophage and monocyte-like cells from rainbow trout, a possible survival strategy. J. Cell. Biochem. 108: 631-637.

Ricardo JL, Carlos RO, Douglas M, Priscila AF, et al. (2007). In vitro cytotoxicity and antioxidant activity of Agaricus subrufescens extracts. Afr. J. Biotechnol. 6: 1144-1150.

Rodríguez NE, Gaur U and Wilson ME (2006). Role of caveolae in Leishmania chagasi phagocytosis and intracellular survival in macrophages. Cell. Microbiol. 8: 1106-1120.

Roop RM 2nd, Gaines JM, Anderson ES, Caswell CC, et al. (2009). Survival of the fittest: how Brucella strains adapt to their intracellular niche in the host. Med. Microbiol. Immunol. 198: 221-238.

Ryckaert J, Bossier P, D’Herde K, Diez-Fraile A, et al. (2010). Persistence of Yersinia ruckeri in trout macrophages. Fish Shellfish Immun. 29: 648-655.

Thirunavukkarasu C, Wang LF, Harvey SA, Watkins SC, et al. (2008). Augmenter of liver regeneration: An important intracellular survival factor for hepatocytes. J. Hepatol. 48: 578-588. 
Wand ME, Müller CM, Titball RW and Michell SL (2011). Macrophage and Galleria mellonella infection models reflect the virulence of naturally occurring isolates of B. pseudomallei, B. thailandensis and B. oklahomensis. BMC Microbiol. 11: 1-11.

Woo SR, Sotos J, Hart AP, Barletta RG, et al. (2006). Bovine monocytes and a macrophage cell line differ in their ability to phagocytose and support the intracellular survival of Mycobacterium avium subsp. paratuberculosis. Vet. Immunol. Immunopathol. 110: 109-120.

Yi JB, Chen Q, Zou WZ, Yan QP, et al. (2008). Starvation effects on pathogenic Vibrio alginolyticus in natural seawater. Acta Oceanol. Sin. 27: 120-129.

Zhao LL, Shao CW, Liao XL, Ma HY, et al. (2009). Twelve novel polymorphic microsatellite loci for the Yellow grouper (Epinephelus awoara) and cross-species amplifications. Conserv. Genet. 10: 743-745. 